\title{
LA FALTA DE EDUCACIÓN FINANCIERA Y SU INCIDENCIA EN LA POBREZA EN LOS MONTES DE MARÍA*
}

\author{
Effects of the lack of financial education on poverty in the \\ Montes de María Region
}

\section{David de Jesús Ardila Leiva1 Gabriel Medina Vergara²}

\section{RESUMEN}

La presente investigación tiene como objeto establecer la incidencia de la falta de educación financiera como factor de pobreza en las familias de las zonas vulnerables del municipio de San Juan Nepomuceno, Bolívar, Colombia. Se realiza el estudio de los conocimientos en finanzas y el uso adecuado de las mismas en sectores deprimidos de este municipio, a través, de una encuesta que determinó las debilidades financieras de estas familias. Así mismo, la interrelación del factor estudiado con un sistema complejo de fenómenos socioeconómicos como el desempleo, la educación básica, la exclusión financiera, entre otros. Lo anterior, permitió concluir las posibles incursiones de modelos ya establecidos en diferentes países de educación financiera, unido a políticas de inclusión y educación financiera promovida por diferentes organismos nacionales e internacionales, cuyo objeto es la disminución de la pobreza y que pueden ser aplicables al caso de la población anteriormente mencionada.

Palabras clave: Educación financiera, pobreza, microfinanzas, inclusión financiera, calidad de vida.

Clasificación JEL: D14, D63, G28, I31

\footnotetext{
* Documento presentado en las Jornadas de Investigación de la Facultad de Ciencias Económicas de la Universidad de Cartagena

1 Programa de Administración Financiera, Facultad de Ciencias Económicas - Universidad de Cartagena. E-mail: david27121996@hotmail.com

${ }^{2}$ Tutor de la Universidad de Cartagena - Centro Tutorial San Juan Nepomuceno. E-mail: gabrie0830@ hotmail.com
} 


\begin{abstract}
This research aims to establish the incidence of the lack of financial education as a factor of poverty in families of vulnerable areas in the municipality of San Juan Nepomuceno, Bolivar, Colombia. The study of knowledge in finance and proper use of them in depressed areas of this municipality is done through of a survey that determined the financial weaknesses of these families. It also studied the interrelation of a complex system of socioeconomic phenomena such as unemployment, basic education, financial exclusion, among other factors. This allowed to conclude the possible incursions of models already established in different countries in financial education, coupled with policies of inclusion and financial education promoted by various national and international organizations, aimed at reducing poverty and that may be applicable to the case of the above population.
\end{abstract}

Keywords: Financial education, poverty, microfinance, financial inclusion, quality of life.

JEL Clasification: D14, D63, G28, I31.

\title{
Introducción
}

El analfabetismo financiero se ha convertido en una preocupación del sistema financiero global, transcendiendo al sector educativo y a las políticas públicas; las consecuencias de este fenómeno se dan a causa de la exclusión financiera, social y económica a la que se ve sometida gran parte de la población mundial y que se evidencia con mayor impacto en los países subdesarrollados y en vía de desarrollo.

En los últimos años han surgido distintos organismos internacionales con el objetivo de combatir la pobreza a través de la educación e inclusión financiera, una de estas es la Organización para la Cooperación y Desarrollo ${ }^{3}$ (OCDE) quien en 2012 se dio a la tarea de medir los niveles de educación financiera en 18 países (entre esos Colombia), a través, del Programe for International Student Assessment (PISA).

En el informe PISA se observa que Colombia ocupa el último lugar con una puntuación de 379, muy por debajo del resto de naciones que hicieron parte del estudio (OECD, 2014); En cuanto a educación financiera "no ha sido medido hasta ahora que tan efectiva es en el bienestar de una persona,

\footnotetext{
${ }^{3}$ Fundada en 1961, la Organización para la Cooperación y el Desarrollo Económicos (OCDE) agrupa a 34 países miembros y su misión es promover políticas que mejoren el bienestar económico y social de las personas alrededor del mundo.
} 
lo que obliga a preguntarse hasta dónde puede llegar el impacto de estas enseñanzas en las decisiones financieras de los colombianos" (Ocampo Lozano, 2014, pág. 182)

El municipio de San Juan Nepomuceno pertenece a la subregión de los Montes de María en el Caribe colombiano; en esta zona los niveles de pobreza alcanza un porcentaje del 43\%; los niveles de educación e inclusión financiera en el municipio se desconocen; sin embargo, es notable la falta de cultura financiera en la forma de vida los habitantes, el mayor porcentaje de éstos no posee conocimientos básicos sobre técnicas adecuadas en el manejo de sus finanzas personales, lo que se refleja directamente en la economía familiar.

Ahora bien; visto desde la óptica de un fenómeno socioeconómico como la pobreza, puede considerarse a la falta de educación financiera como un talante influyente en los índices del mismo; teniendo en cuenta que ésta es una de las principales problemáticas que afectan el municipio de San Juan Nepomuceno, se reconoce esta problemática como un riesgo directo en la economía familiar.

Por lo anterior esta investigación pretende determinar la influencia de la falta de educación financiera como un factor de pobreza en las familias del municipio en cuestión, por lo cual se hace necesario establecer las causas y consecuencias de esta problemática además de establecer las debilidades financieras de los individuos que conforman esta población con el fin de plantear diferentes estrategias de formación básica que permitan mitigar este flagelo de desconocimiento en finanzas.

El presente artículo se divide en seis secciones; en primer lugar se encuentra el marco teóricoempírico que da soporte a la investigación, en segundo lugar se presenta la metodología que conduce la investigación, la cuarta sección muestra los resultados y la discusión de los mismo, en una quinta instancia se presentan las conclusiones y recomendaciones del estudio, en la sexta sección se haya la lista de referencias bibliográficas mencionadas en el cuerpo del escrito y finalmente se exhiben los anexos correspondientes.

\section{Marco teórico-empírico}

La OCDE conceptualiza a la educación financiera como el:

Proceso por el cual los consumidores/inversionistas financieros mejoran su comprensión de productos financieros, los conceptos y los riesgos, y, mediante información, instrucción y/o 
asesoramiento objetivo, logran desarrollar habilidades y confianza para ser más conscientes de los riesgos y oportunidades financieras, tomar decisiones informadas, saber a dónde ir para obtener ayuda y ejercer cualquier acción eficaz para mejorar su bienestar económico (OECD, 2005).

Frente a esto Ocampo (2014), argumenta que:

La educación financiera no es una materia que simplemente se aprenda y se memorice, es una herramienta tan dinámica como la economía misma de un país y la situación financiera de una persona, de forma tal que el estar educado financieramente le permite a un individuo ser consciente de las decisiones que impactan diferentes etapas de su vida y por ende como él se la relaciona con un sector y como aporta a la economía de un país según la asertividad de sus decisiones en función a lo aprendido en esta materia (Ocampo Lozano, 2014, págs. 188, 189).

El Banco Mundial en 2015 presenta un informe en el que se da a conocer una panorámica del nivel de educación financiera de la población en general a escala mundial como se observa en el gráfico 1. (López del Paso, 2015), presenta los resultados de este informe y expresa que:

El informe se basa en una encuesta realizada por teléfono en 2014 a más de 140.000 adultos (mayores de 15 años) de 140 nacionalidades, conformada por cuatro cuestiones básicas sobre diversificación del riesgo, inflación y tipo de interés simple y compuesto. Atendiendo a la metodología empleada, se entiende que una persona dispone de un nivel de conocimiento adecuado cuando responde correctamente a 3 de las 4 preguntas planteadas. De acuerdo con los resultados obtenidos, sólo el 33\% de la población mundial supera este umbral (pág. 1).

Gráfico 1. Nivel de educación financiera en el mundo. 2014 Porcentaje de adultos que superan 3 de las 4 cuestiones planteadas

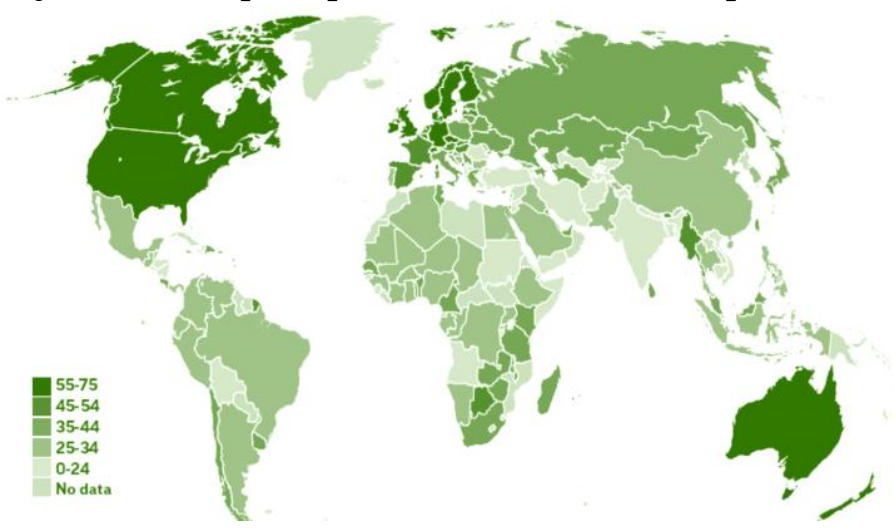

Fuente: Banco Mundial 
Por otro lado; la inclusión financiera engloba en su práctica a la de educación financiera, en cuanto a este tema Cull, Ehrbeck, \& Holle, (2014) y Sanchez \& Rodriguez, (2015) afirman que:

Los pobres están excluidos, generalmente, de las oportunidades de empleo asalariado que presupone la teoría económica tradicional. Viven y trabajan en la economía informal, no por elección sino por necesidad. En términos económicos, son, al mismo tiempo, hogares que consumen y empresas cuentapropistas; por lo tanto, las decisiones en materia de consumo y producción están interrelacionadas. En consecuencia, necesitan un amplio espectro de servicios financieros para crear y mantener medios de subsistencia, acumular activos, gestionar los riesgos y ordenar el consumo. Con frecuencia, la distinción tradicional entre las necesidades financieras de los consumidores y las de las empresas se desdibuja (Cull et al., 2014, pág. 1).

Los programas de educación financiera dirigidos a la población excluida pueden ser, en muchos casos, una buena forma de mejorar el conocimiento que las entidades financieras tienen sobre el fenómeno de la exclusión financiera. Gracias a la información recibida de destinatarios finales y entidades, colectivos o instituciones con los que colaboran, aquéllas pueden mejoran su comprensión acerca del sector de población excluido (habitualmente no bancarizado) (Sánchez Macías \& Rodriguez López, 2015, pág. 20).

Dentro de la educación financiera las microfinanzas juegan un papel fundamental; un informe titulado: "Últimas conclusiones derivadas de estudios de evaluación aleatorios de las microfinanzas", realizado por Bauchet, Marshall, Starita, Thomas, \& Yalouris, (2011) para el CGAP y sus asociados, presenta una recopilación de diferentes estudios sobre microfinanzas y concluyen que:

El trabajo de investigadores y proveedores de microfinanzas participantes ofrece nuevos conocimientos sobre la manera en que los clientes utilizan el capital, sobre lo que los ayuda a ahorrar, y sobre las restricciones que enfrentan y que les impiden obtener mayores beneficios del acceso al financiamiento (Bauchet et al., 2011, pág. 21).

Rosenberg, (2010) por su parte también realiza una agrupación de estudios sobre el impacto del microcrédito en la pobreza; en éste expone que:

La pobreza económica no es solo un problema de ingresos bajos, también es un problema de ingresos irregulares e inciertos. Para poder llevar la comida a la mesa todos los días y para satisfacer 
otras necesidades de consumo básicas, los hogares pobres tienen que ahorrar y pedir prestado constantemente. (Rosenberg, 2010, pág. 2).

El Fondo Multilateral de Inversiones ${ }^{4}$ (FOMIN) en uno de sus informes elaborado por (Trujillo \& Navajas, 2014) y en el que se evidencia el panorama de inclusión financiera para Latinoamérica y el Caribe, presenta entre sus hallazgos lo siguiente:

1. La cartera de microcrédito de la región está alrededor de los 40.000 millones de dólares, considerando tanto entidades del sistema financiero reguladas como no reguladas. Esta cifra que constituye alrededor del $2 \%$ del crédito al sector privado no financiero por parte del sistema financiero regulado. Los clientes en la región superan los 22 millones de personas.

2. Al contrastar estos datos con el total de la $\mathrm{PEA}^{5}$, la población informal en cada país o el número de microempresarios o microempresas, observamos grandes disparidades en los resultados de los diferentes países de la región. Los países que mayor nivel de penetración son Bolivia, Colombia, Ecuador, Perú, México y Chile, y existen países en que los que el nivel de alcance del microcrédito es todavía limitado como Argentina, Uruguay o Venezuela.

3. El análisis del nivel de competencia de estos mercados en la región también presenta una gran variabilidad. De un total de 21 países de la región 13 de ellos presentan altos niveles de concentración de mercado (Colombia, El Salvador o Guatemala, entre otros) mientras que 8 de ellos presentan sectores no concentrados (Ecuador, Honduras o Perú, entre otros). págs. 6,7.

Ocampo (2014) expone que en el caso de Colombia:

El sector financiero viene desde hace varios años desarrollando programas tendientes a educar financieramente a sus clientes y usuarios, esto de alguna manera ha sido el resultado de las políticas implementadas por la Superfinanciera dada la normatividad existente hacia la Protección del Consumidor Financiero, motivado a su vez por el comportamiento mismo de la economía donde tanto crisis como bonanzas han dejado lecciones que aprender (pág 181).

\footnotetext{
${ }^{4}$ Fundado en 1993 como miembro del Grupo Banco Interamericano de Desarrollo, el Fondo Multilateral de Inversiones (FOMIN) fue creado para desarrollar soluciones efectivas que apoyan el crecimiento económico y la reducción de la pobreza a través del desarrollo liderado por el sector privado. Su misión es trabajar como laboratorio de desarrollo, y experimentar, innovar y asumir riesgos con el fin de construir y apoyar modelos exitosos de negocio para las micro, pequeña y mediana empresa. El FOMIN trabaja por medio de donaciones, préstamos e inversiones de capital y es el principal proveedor internacional de asistencia técnica para el sector privado en Latinoamérica y el Caribe.

${ }^{5}$ Los autores hacen referencia a la Población Económicamente Activa (PEA).
} 
Tabla 1. Cartera de microcrédito y clientes de América Latina y el Caribe

\begin{tabular}{|c|c|c|c|c|c|c|c|}
\hline Categorías & $\begin{array}{l}\text { Número de } \\
\text { Instituciones }\end{array}$ & $\%$ & $\begin{array}{c}\text { Cartera de } \\
\text { microcrédito } \\
\text { (millones de US\$) }\end{array}$ & $\%$ & $\begin{array}{c}\text { Número de } \\
\text { Clientes de } \\
\text { microcrédito }\end{array}$ & $\%$ & $\begin{array}{c}\text { Crédito } \\
\text { promedio } \\
\text { (US\$) }\end{array}$ \\
\hline Reguladas & 350 & 33 & 31.742 & 79 & 14.279 .435 & 65 & 2.233 \\
\hline Bancos & 174 & 16 & 24.861 & 62 & 9.648 .913 & 44 & 2.577 \\
\hline No Bancarias & 135 & 13 & 6.581 & 16 & 4.548 .572 & 21 & 1.447 \\
\hline Cooperativas & 41 & 4 & 300 & 1 & 81.950 & 0,4 & 3.663 \\
\hline $\begin{array}{l}\text { No } \\
\text { Reguladas }\end{array}$ & 711 & 67 & 8.291 & 21 & 7.800 .229 & 35 & 1.063 \\
\hline $\begin{array}{l}\text { ONG y otras } \\
\text { formas } \\
\text { institucionales }\end{array}$ & 442 & 40 & 5.665 & 14 & 7.017.205 & 32 & 807 \\
\hline Cooperativas & 289 & 27 & 2.626 & 7 & 783.024 & 4 & 3.353 \\
\hline $\begin{array}{l}\text { Todas las } \\
\text { Instituciones } \\
-2013 \quad(28) \\
\text { países }\end{array}$ & 1.061 & & 40.033 & & 22.079 .664 & & 1.813 \\
\hline $\begin{array}{l}\text { Todas las } \\
\text { Instituciones } \\
-\quad 2012 \quad(28) \\
\text { países }\end{array}$ & 1.085 & & 37.152 & & 20.534 .264 & & 1.839 \\
\hline $\begin{array}{l}\text { Todas las } \\
\text { Instituciones } \\
-2010 \quad(28) \\
\text { países }\end{array}$ & 675 & & 15.168 & & 12.476 .379 & & 1.216 \\
\hline $\begin{array}{l}\text { Todas las } \\
\text { Instituciones } \\
-2005 \quad(28) \\
\text { países }\end{array}$ & 336 & & 5.437 & & 5.952 .716 & & 913 \\
\hline $\begin{array}{l}\text { Todas las } \\
\text { Instituciones } \\
-2001 \quad(28) \\
\text { países }\end{array}$ & 184 & & 1.189 & & 1.806 .445 & & 659 \\
\hline \multicolumn{8}{|c|}{$\begin{array}{l}\text { Notas: } \\
\text { (1) Los datos sobre microcrédito varían año a año por la evolución natural del mercado, la aparición de nuevas fuentes } \\
\text { de información y el uso de nuevas definiciones de microcrédito en la región, por lo tanto hay que ser cautelosos con } \\
\text { las comparaciones interanuales. En algunos países que carecen de definición de microcrédito para fines de supervisión } \\
\text { financiera se ha utilizado la información disponible sobre crédito a la microempresa que normalmente se basa en el } \\
\text { tamaño de la empresa a la que se presta recursos. }\end{array}$} \\
\hline
\end{tabular}

\section{Fuente: FOMIN, BID.}

\section{Metodología}

La investigación se enmarca en un estudio de alcance descriptivo; busca a partir de la información recolectada describir la incidencia de una problemática en cierto factor que centra la línea de investigación llevada a cabo. Es de carácter mixto ya que cumple con características cuantitativas y cualitativas; y no experimental puesto que no se van a manipular las variables. 
La población utilizada en este proyecto son todos los habitantes de las zonas vulnerables (en cuanto a pobreza) del municipio San Juan Nepomuceno (Bolívar), en su mayoría pertenecientes a los barrios aledaños a las riveras que comprenden los arroyos rastro, catalina y el salvador (ver tabla de 2 . Distribución muestral).

Tabla 2. Distribución muestral

\begin{tabular}{|c|c|}
\hline Barrio & $\begin{array}{c}\text { Muestra } \\
\text { (Familias) }\end{array}$ \\
\hline 23 de mayo & 10 \\
\hline Pueblo Nuevo & 10 \\
\hline El Ojito & 10 \\
\hline Porqueritas & 10 \\
\hline Las Delicias & 10 \\
\hline Cerrito & 10 \\
\hline 20 de marzo & 10 \\
\hline Palmira & 10 \\
\hline Total, Muestra & $\mathbf{8 0}$ \\
\hline
\end{tabular}

Fuente: Elaboración propia

Teniendo en cuenta la línea de investigación escogida; se utilizan las siguientes técnicas de recolección de información:

- Observación del entorno socio-económico.

- Cuestionario con preguntas semi-abiertas y cerradas (ver anexo 3).

Encaminadas a la búsqueda de información veraz y sustancial que permita dar respuesta a las preguntas específicas planteadas en un principio con el fin de dar cumplimiento a los objetivos trazados. 


\section{DISCUSIÓN DE RESULTADOS}

\section{Desconocimiento del concepto de educación financiera}

En el estudio realizado se logró establecer que el 97,75\% (gráfico 3) de los encuestados no tiene una idea clara del concepto de educación financiera, a pesar de lo que se observa en el gráfico 2 en donde el $36,25 \%$ de la muestra dio una opinión de lo que supone es la educación financiera.

Gráfico 2. ¿Para usted qué es Educación Financiera?

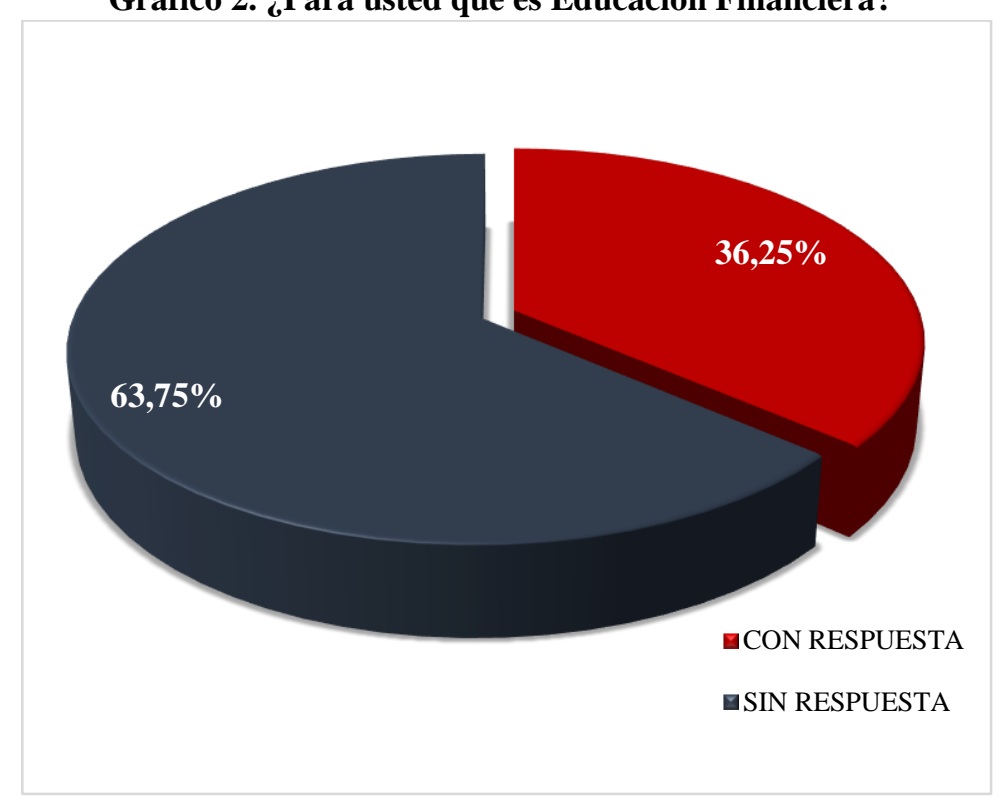

Fuente: Elaboración propia con resultados de la encuesta realizada a los habitantes de los sectores vulnerables del municipio de San Juan Nepomuceno (Bolívar).

Gráfico 3. Correcto conocimiento del concepto de Educación Financiera.

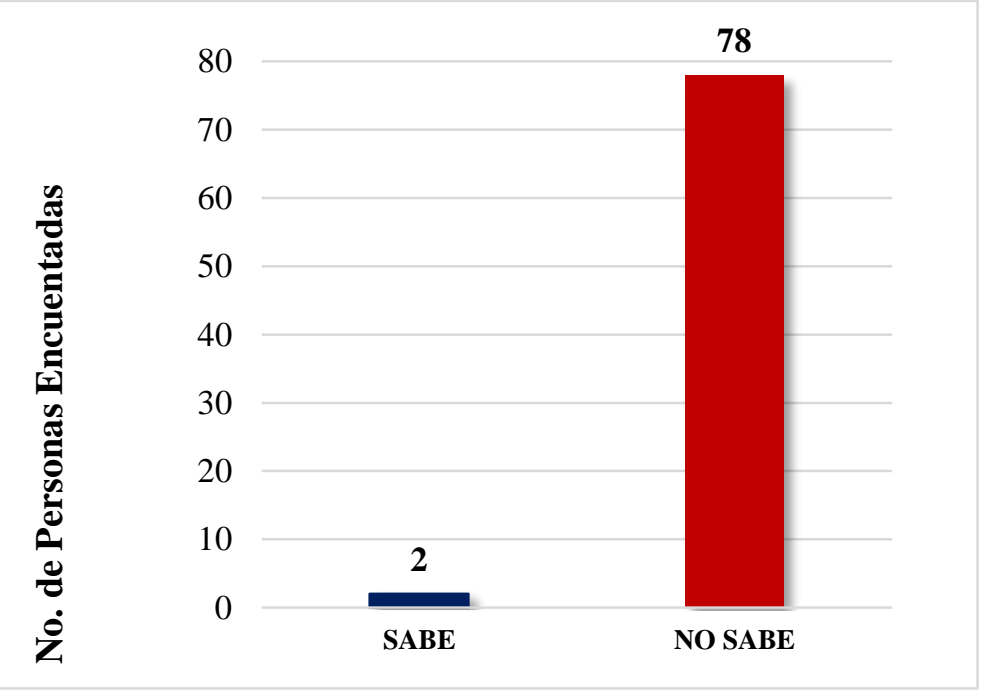

Fuente: Elaboración propia con resultados de la encuesta realizada a los habitantes de los sectores vulnerables del municipio de San Juan Nepomuceno (Bolívar) 


\section{Capacitación y asesoría financiera}

Existe un porcentaje cuantioso de la población el cual nunca ha recibido asesoría para la optimización de sus finanzas familiares $(78,75 \%)$ como se aprecia en el gráfico 4 . Quienes alguna vez recibieron orientación sobre sus finanzas $(21,25 \%)$ en su mayoría se desempeñan como mujeres cabeza de hogar y lo han hecho a través de pequeños talleres o cursos del Servicio Nacional de Aprendizaje (SENA), del Departamento para la Prosperidad Social (DPS), en sus trabajos, en el Programa Familias en Acción, entre otros.

Fuente: Elaboración propia con resultados de la encuesta realizada a los habitantes de los sectores vulnerables del municipio de San Juan Nepomuceno (Bolívar).

Así mismo se pudo determinar que la falta de educación financiera es a causa de la carencia de orientación e interés por parte del gobierno o de entidades públicas y privadas para sensibilizar a la comunidad y brindar asesoría sobre el manejo de sus finanzas familiares. Cabe resaltar que, si bien han existido algunos programas para distintos sectores de la población en materia de educación financiera, éstos no han sido eficaces en el cumplimiento de su objeto, por tanto, la problemática subsiste; además de la falta de iniciativa propia, producto de la desinformación a la que se ven sometidas estas familias.

\section{Cubrimiento de necesidades básicas}

Según el estudio al 53,75\% de la población en cuestión no le alcanza sus ingresos para suplir las necesidades básicas de su familia, destacando como principal razón el alto costo de vida, la falta de empleo, salarios insuficientes, entre otros (ver tabla 3). Mientras el resto $(46,25 \%)$ como se presenta en el gráfico 5, afirman que sí le alcanza debido a que se abstienen a gastar de más y suplen sus necesidades más básicas con el dinero que ingresa al hogar. 
Gráfico 5. ¿Le alcanzan sus ingresos para suplir las necesidades básicas de su familia?

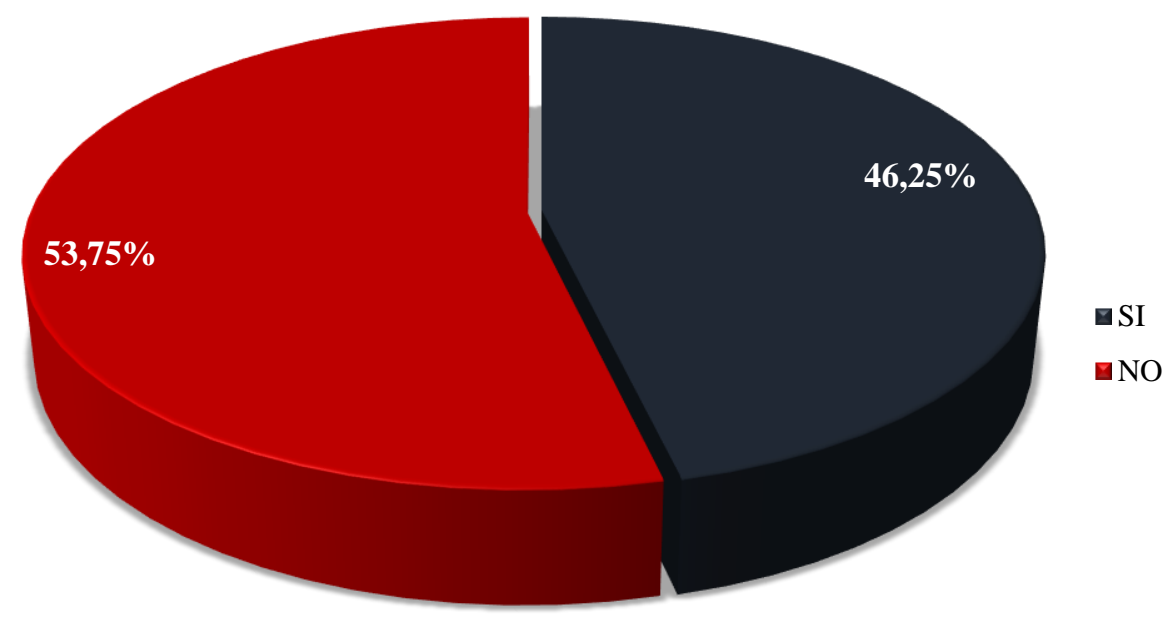

Fuente: Elaboración propia con resultados de la encuesta realizada a los habitantes de los sectores vulnerables del municipio de San Juan Nepomuceno (Bolívar).

Tabla 3. Principales razones para el cubrimiento o no de las necesidades básicas

\begin{tabular}{|c|c|}
\hline RESPUESTA & PRINCIPALES RAZONES \\
\hline SI & $\begin{array}{l}\text { - Porque sabe administrar el dinero. } \\
\text { - Porque hay educación, alimentación y no le falta nada. } \\
\text { - } \text { Porque lucha para que el dinero le alcance. } \\
\text { - } \text { Pe dividen bien los recursos. } \\
\text { - Porque cubre sus necesidades más básicas. } \\
\text { - Porque gasta solo lo necesario. } \\
\text { - Porque lo más importante es la alimentación y salud y la tiene. } \\
\text { - Porque gana poco, pero está conforme. } \\
\text { - Porque su sueldo cubre todos sus gastos. }\end{array}$ \\
\hline NO & $\begin{array}{l}\text { - Porque no le alcanza el dinero para comprar las cosas necesarias. } \\
\text { - Porque la canasta familiar ha incrementado mucho de precio. } \\
\text { - Porque no ingresa suficiente dinero. } \\
\text { - Porque no hay trabajo. } \\
\text { - Solo trabaja el esposo y los servicios están costosos. } \\
\text { - Porque lo que gana su esposo solo cubre la alimentación. } \\
\text { - Porque es campesino y van dos años que no ha cosechado. } \\
\text { - Porque solo realiza algunos empleos momentáneos para cubrir } \\
\text { - } \text { Porqunas necesidades. }\end{array}$ \\
\hline
\end{tabular}

Fuente: Elaboración propia con resultados de la encuesta realizada a los habitantes de los sectores vulnerables del municipio de San Juan Nepomuceno (Bolívar). 


\section{Acceso al crédito informal}

Uno de los aspectos identificados es la proporción del 41,25\% que afirmó, recurre a créditos informales a altas tasas de interés o en la "modalidad gota a gota"6 para poder subsistir (ver gráfico 6). Esta práctica además de ser un delito se convierte en un peligro incluso para la vida de quien se ven sometidos a esta práctica, algunas veces, cuando quien obtiene el crédito no puede cumplir con el pago, puede ser víctima de agresiones verbales o físicas de parte de quien otorga el crédito o en su defecto del “cobrador" encargado.

Gráfico 6. Personas que recurren a créditos informales para subsistir

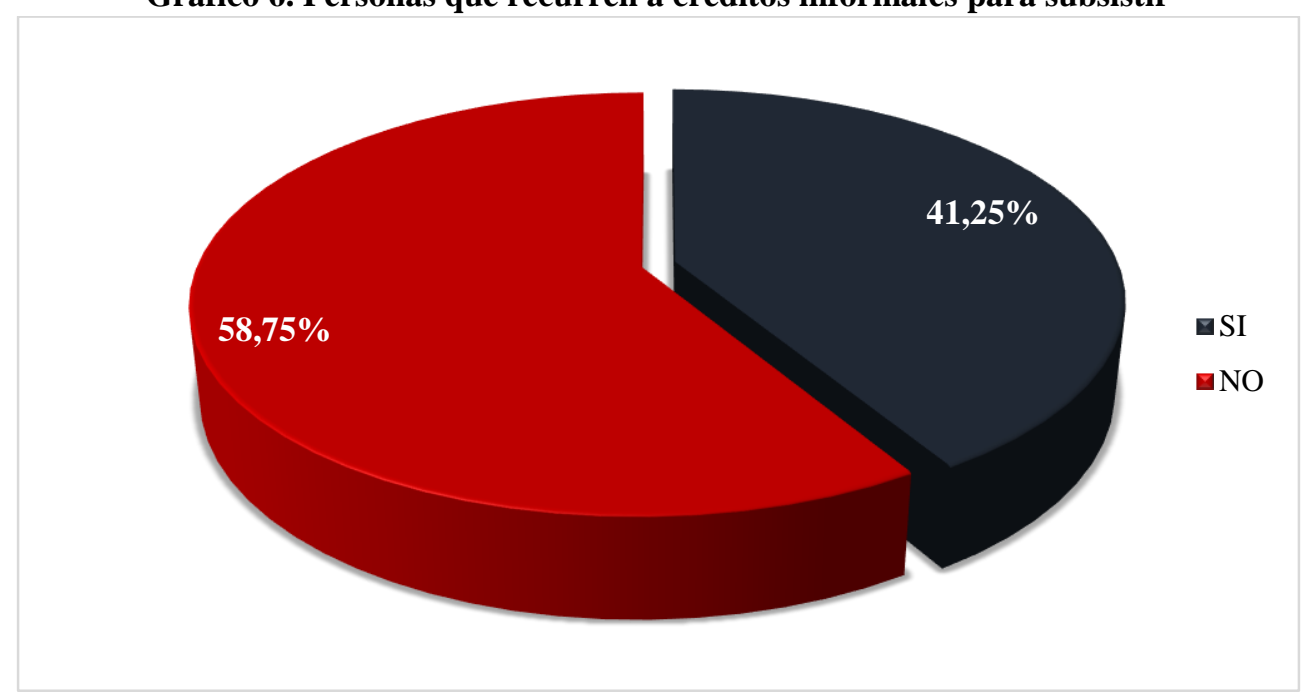

Fuente: Elaboración propia con resultados de la encuesta realizada a los habitantes de los sectores vulnerables del municipio de San Juan Nepomuceno (Bolívar).

\section{Necesidades en educación financiera}

Las necesidades en materia de educación financiera que poseen las familias de las zonas vulnerables del municipio de San Nepomuceno están relacionadas a la falta de ahorro, manejo de las deudas, elaboración del presupuesto familiar e inversión (ver gráfico 7). Por lo que es imperativo implementar de estrategias de formación financiera básica que ayuden a cubrir las falencias en el manejo que le dan a su economía estas familias.

\footnotetext{
${ }^{6}$ perteneciente al mercado extrabancario y contemplada como un delito por el código penal (artículo 305), en el cual se establece que incurren en el delito de usura quien reciba o cobre dinero, directa o indirectamente, a cambio de préstamos o por concepto de venta de bienes o servicios a plazos, y que obtengan una utilidad o ventaja que exceda la mitad del interés bancario corriente.
} 
Gráfico 7. Práctica de los principios de la gestión optima de las finanzas personales

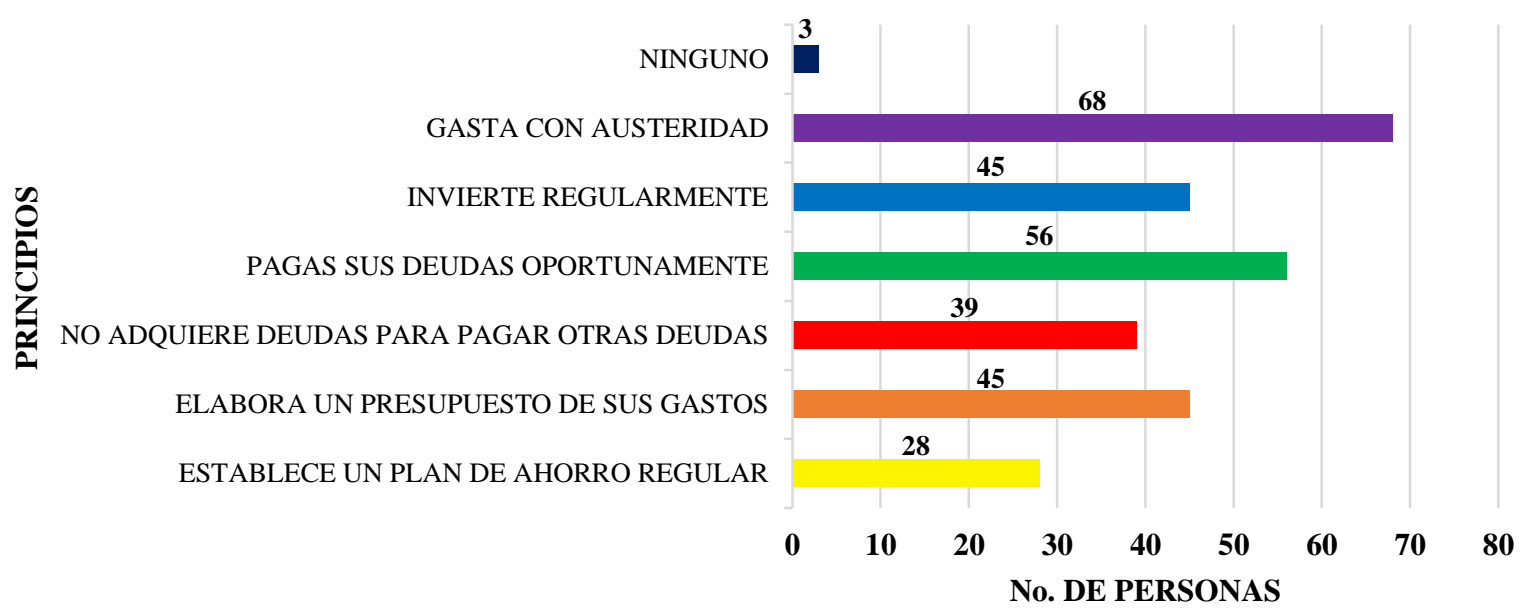

Fuente: Elaboración propia con resultados de la encuesta realizada a los habitantes de los sectores vulnerables del municipio de San Juan Nepomuceno (Bolívar).

\section{Disponibilidad a recibir Educación Financiera}

Uno de los resultados del estudio que llama la atención, es la disponibilidad a recibir educación financiera por parte de casi la totalidad de los encuestados $(98,75 \%)$, pues suponen que esto contribuiría a mejorar su calidad de vida y la de los miembros de su hogar (ver gráfico 8).

Gráfico 8. ¿Le gustaría recibir educación financiera?

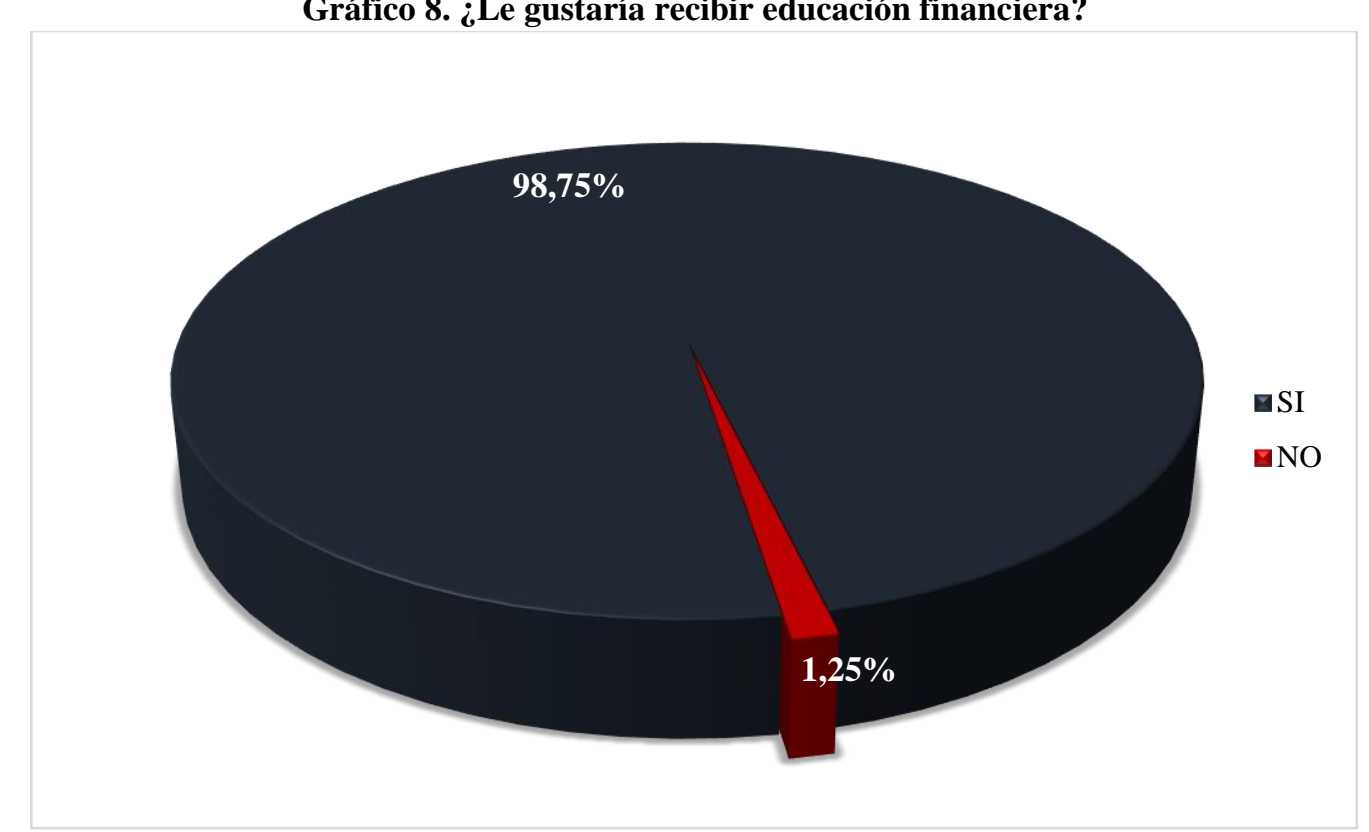

Fuente: Elaboración propia con resultados de la encuesta realizada a los habitantes de los sectores vulnerables del municipio de San Juan Nepomuceno (Bolívar). 


\section{Percepción sobre la educación financiera como mitigadora de la pobreza}

Del total de la muestra estudiada el 93,75\% (ver gráfico 9), manifestó que considera es importante la educación financiera para disminuir la brecha de pobreza que existe en el municipio. La mayoría considera que una de las formas de disminuir el índice es que las familias aprendan a manejar adecuadamente sus recursos y lo cual debe ir de la mano de estrategias que fomenten la creación de nuevos empleos.

Gráfico 9. ¿Considera importante la educación financiera para mitigar la pobreza?

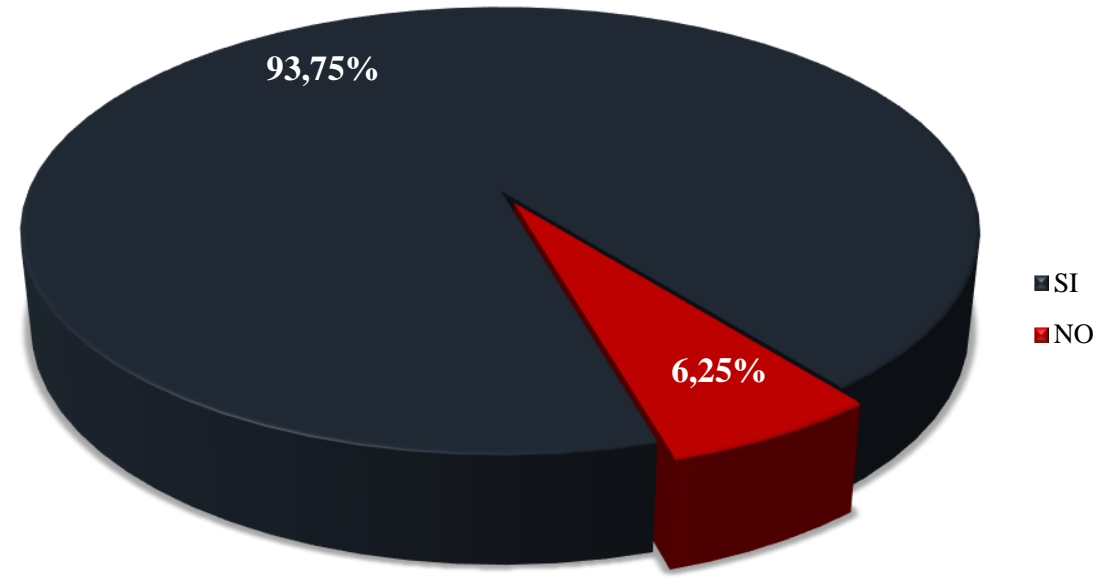

Fuente: Elaboración propia con resultados de la encuesta realizada a los habitantes de los sectores vulnerables del municipio de San Juan Nepomuceno (Bolívar).

\section{Apoyo a la creación de espacios de Educación Financiera y Emprendimiento}

Por último; uno de los resultados más significativos de esta investigación es el apoyo que brindaría el 100\% de la población a la creación de un centro de educación financiera donde se le brinde asesoría a las familias y particulares sobre el manejo de sus finanzas complementado con la incentivación y orientación de proyectos productivos que brinden fuentes de empleo precisamente para la población en situación de vulnerabilidad del municipio (ver gráfico 10). 
Gráfico 10. ¿Apoyaría la creación de un centro de Educación Financiera y Emprendimiento?

Fuente: Elaboración propia con resultados de la encuesta realizada a los habitantes de los sectores vulnerables del municipio de San Juan Nepomuceno (Bolívar).

\section{CONCLUSIONES}

La educación financiera permite que las personas tanto de forma individual como familiar puedan manipular de manera óptima sus recursos; desafortunadamente en un gran volumen de naciones este tipo de educación es casi nulo. Para el caso de Colombia la brecha de educación financiera es amplia y los mecanismos hasta ahora utilizados no han sido eficaces.

Ahora bien; está comprobado que el fenómeno de pobreza está ligado a los niveles de educación e inclusión financiera, se ha convertido en tarea de diversos organismos internacionales (OCDE, Alianza para la Inclusión Financiera, Banco Mundial, entre otros.) luchar por el desarrollo y bienestar de las masas, a través, de múltiples programas y estrategias.

En el municipio de San Juan Nepomuceno, Departamento de Bolívar, Colombia; el término educación financiera se considera ajeno al léxico de sus habitantes, las malas prácticas financieras unidas a diversos factores socioeconómicos (desempleo, costo de vida, nivel de educativo) conllevan a disminuir la calidad de vida y aumentar los índices de pobreza, sobre todo en los sectores vulnerables. Un porcentaje alto de estas familias recurren a créditos con tasas de interés excesivamente altas, lo cual aseguran es imprescindible para subsistir; se convierte entonces en un círculo vicioso de endeudamiento, que nunca termina y cada día crece más. 
Así mismo, se evidencia que no existen fuentes de empleo; el sector económico primario cada día disminuye y la presencia del sector secundario es casi nulo, el factor producción al estar alejado de la economía municipal afecta directamente a las familias desmejorando sus ingresos.

Por tal motivo se evidencia la disponibilidad de las familias para que sus miembros sean educados financieramente; se convierte en una tarea de profesionales de las finanzas, sector bancario, sector educativo y gobierno local la implementación de los respectivos programas y estrategias que permitan dar fin a la problemática. Es sabido que no es tarea fácil erradicar la pobreza solo con educar en finanzas a la población, pero sin duda es un gran paso hacia la consecución de este objetivo.

Entre las diversas estrategias a formular se recomienda tomar como referencia la propuesta de la Massive Open Online Course (MOOC) al cual hace referencia (Domínguez Martínez, 2014). La propuesta de MOOC consiste en un curso de doce módulos, con los objetivos de "adquirir conocimientos en materia de finanzas, saber utilizarlos en beneficio propio y realizar elecciones informadas, de manera responsable y consciente" (pág. 80).

Otra estrategia adaptable es el material de educación financiera impartido en España por la (FUNDACIÓN EXIT, 2010), consiste en un taller teórico práctico cuya duración es de un mes para aprender temas relacionado con el manejo de gastos y el presupuesto familiar.

De esta forma se avanza en la construcción de una sociedad educada financieramente y se provee la garantía de reducción en índices de pobreza para esta población; Aún queda por resolver la

problemática de desempleo y falta de emprendimiento los cuales se establecen como los siguientes temas a desarrollar finalizada la presente investigación.

\section{REFERENCIAS BIBLIOGRAFICAS}

Bauchet, J., Marshall, C., Starita, L., Thomas, J., \& Yalouris, A. (2011). Últimas conclusiones derivadas de estudios de evaluación aleatorios de las microfinazas. Informes del CGAP y sus Asociados No. 2. 
Castro-Alfaro, A., Mercado-León, L., Gonzalez-Pérez, N. (2016). Responsabilidad social corporativa en cadenas hoteleras: Hotel Las Américas en Cartagena de Indias. Panorama Económico, 24, 283-296 DOI: https://doi.org/10.32997/2463-0470-vol.24-num.0-2016-1563

Canaviri-Sillerico, G., Aliaga-Lordemann, F. (2016). Construcción de un indicador de pobreza multidimensional mediante análisis combinatorio. Panorama Económico, 24. 85-104. DOI: https://doi.org/10.32997/2463-0470-vol.24-num.0-2016-1551

Clavijo-Vera, S., Vera-Sandoval, A., Vera-Concha, N. (2015). Politica monetaria: reglas y discreción. Panorama Económico, 23: 31-38. DOI: https://doi.org/10.32997/2463-0470-vol.23-num.12015-1375

Cull, R., Ehrbeck, T., \& Holle, N. (2014). La inclusión financiera y el desarrollo: Pruebas recientes de su impacto. Enfoques, No. 29, 1.

De la Hoz, A., Camargo-Montoya, P., Saldarriaga-Arango, C., Praj, D. (2014). Análisis de los riesgos en las importaciones colombianas. Panorama Económico, 22: 63-75. DOI: https://doi.org/10.32997/2463-0470-vol.22-num.0-2014-1360

Domínguez Martínez, J. (2014). Educación Financiera para la ciudadanía: una propuesta de MOOC. eXtoikos(15).

Flores-Tapia, C., Flores-Cevallos, K. (2017). Impactos económicos inducidos por la demanda final en sectores estratégicos para el cambio de la estructura productiva del Ecuador. Panorama Económico, 25, 3: 443-458. DOI: https://doi.org/10.32997/2463-0470-vol.25-num.3-2017-2085

Fundación Éxito (2010). Material de Educación Financiera. Barcelona, España. Recuperado el 2016, de www.edufinanciera.edu.com 
García-García, F. (2017). Debate sobre la inclusión del cooperativismo dentro de las políticas de la nueva ruralidad en América Latina. Panorama Económico, 25, 3: 357-380. DOI: https://doi.org/10.32997/2463-0470-vol.25-num.3-2017-2081

López del Paso, R. (2015). Nivel de educación financiera en el mundo en 2014. EXtoikos (No. 17), 1-2.

Missaglia, M. (2015). ¿Se pueden aplicar las ideas Keynesianas Al largo plazo? unas reflexiones teóricas y un modelo ilustrativo. Panorama Económico, 23: 17-30. DOI: https://doi.org/10.32997/2463-0470-vol.23-num.1-2015-1380

Ocampo Lozano, M. (2014). Sistema financiero colombiano y la educación financiera. Caso Davivienda. Aglala, 181.

OECD. (2005). Improving Financial Literacy: Analysis of issues and policies. Paris: OECD Publishing. Obtenido de OECD.

OECD. (2014). PISA 2012 Results: Students and Money: Financial Literacy Skills for the 21st Century. PISA, OECD publishing, VI.

Passos-Simancas, E., Arias-Aragones, F. (2016). El capital humano como factor de competitividad en la industria hotelera y turística en Cartagena. Panorama Económico, 24, 269-282. DOI: https://doi.org/10.32997/2463-0470-vol.24-num.0-2016-1561

Rosenberg, R. (2010). ¿El microcrédito ayuda realmente a los pobres? Enfoques (No. 59), 2.

Salazar-Mosquera, G. (2017). Factores determinantes del desempeño financiero en el sector manufacturero en la República del Ecuador. Panorama Económico, 25, 2: 243-254. DOI https://doi.org/10.32997/2463-0470-vol.25-num.2-2017-2076

Sánchez Macías, J. I., \& Rodriguez López, F. (2015). Educación financiera para la inclusión financiera: una hoja de ruta. eXtoikos, No. 17, 20. 
Trujillo, V., \& Navajas, S. (2014). Inclusión financiera en América Latina: Datos y tendencias. FOMIN, BID, Diciembre, 2014.

\section{ANEXOS}

Anexo 1. Desempeño en educación financiera

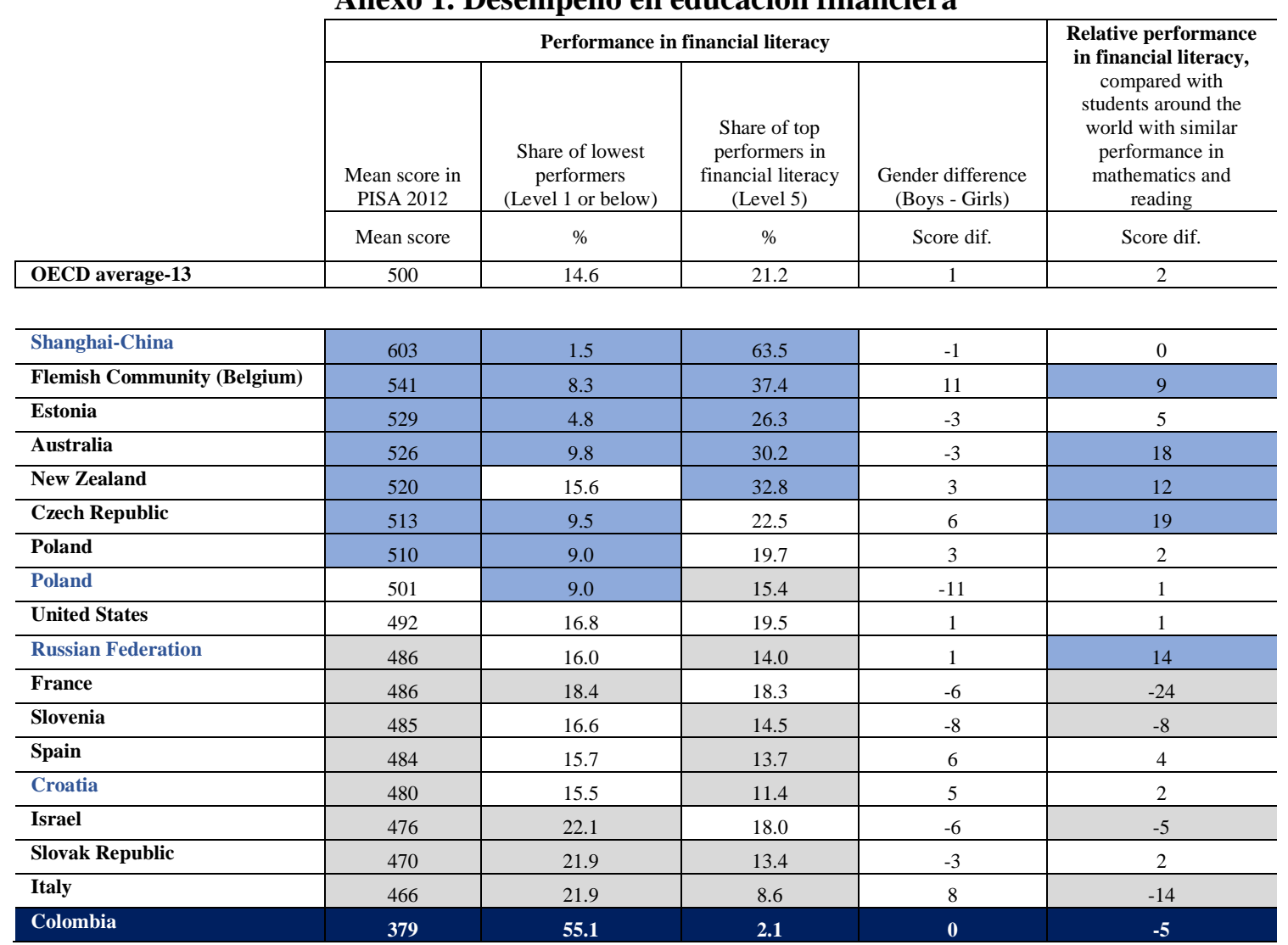

Fuente: OECD, PISA 2012 Database, Tables VI.2.1, VI.2.2, VI.2.4 and VI.3.2.

DOI: $\underline{\text { http://dx.doi.org/10.1787/888933094944 }}$

Anexo 2. Nivel de educación financiera y desarrollo económico. 2014

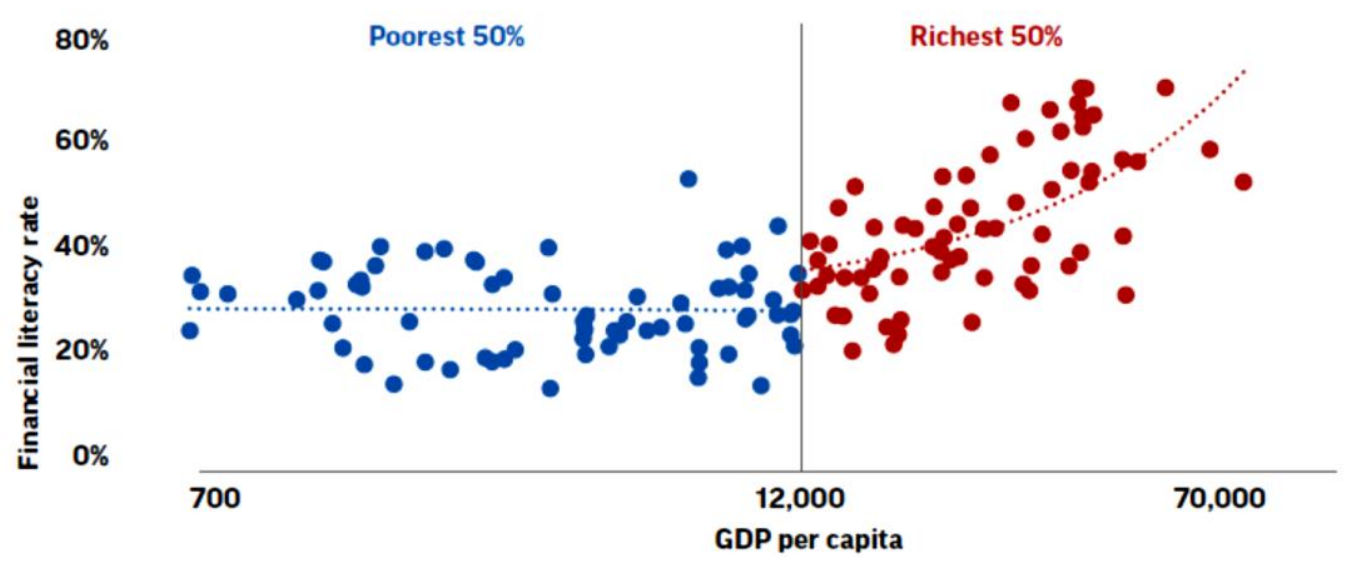

Fuente: Banco Mundial (2015) 


\section{Anexo 3. Formato de Recolección de Información Primaria}

\section{ENCUESTA}

EDAD: SEXO: Masculino $\square$ Femenino $\square$ BARRIO:

1. ¿Para usted qué es la educación financiera?

2. ¿Ha recibido alguna vez asesoría sobre sus finanzas personales o familiares para optimizar el manejo de estas? SI NO en caso de responder SI ¿qué tipo de asesoría fue?

3. ¿Le alcanzan sus ingresos para suplir las necesidades básicas de su familia? SI NO ¿Por qué?

4. ¿Se ve usted en la necesidad de realizar créditos informales a altas tasas de interés para subsistir? SI $\mathrm{NO}$

5. A continuación se presentan algunos principios para el buen manejo de sus finanzas personales y familiares, marque con una $\mathbf{X}$ si pone en práctica alguno de estos; de lo contrario marque en la casilla NINGUNO.

Establece un plan de ahorro regular

Elabora un presupuesto de sus gastos

No adquiere deudas para pagar otras deudas

Paga sus deudas oportunamente

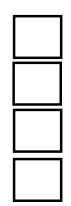

Invierte Regularmente

Gasta con austeridad

Ninguno

6. ¿Le gustaría que su familia recibiera educación financiera para optimizar el uso de sus ingresos y mejorar la calidad de vida de quienes conforman su hogar? SI___ NO___ ¿Por qué?

7. ¿Cree usted qué es importante la educación financiera para disminuir la brecha de pobreza que hay en el municipio? SI NO ¿Por qué?

8. ¿Apoyaría usted la creación de un centro de educación financiera donde le brinden asesoría sobre el buen manejo de sus finanzas personales y familiares; además de incentivar y orientar el desarrollo de proyectos productivos que brinden fuentes de trabajo y crecimiento de la economía del municipio? SI $\mathrm{NO}$ 